\title{
МОНИТОРИНГ УРОВНЯ ТИРЕОГЛОБУЛИНА ВЫСОКОЧУВСТВИТЕЛЬНЫМ МЕТОДОМ У ПАЦИЕНТОВ ПОСЛЕ ЛЕЧЕНИЯ ДИФФЕРЕНЦИРОВАННОГО РАКА ЩИТОВИДНОЙ ЖЕЛЕЗЫ
}

\author{
Маколина Н.П., Платонова Н.М., Трошина Е.А., Мельниченко Г.А. \\ ФГБУ «НМИЦ эндокринологии» Минздрава России, Москва
}

\begin{abstract}
ВВЕДЕНИЕ: современная стратегия наблюдения за больными после радикального лечения дифференцированного рака щитовидной железы (ДРЩЖ) базируется на динамическом мониторинге уровня сывороточного тиреоглобулина (ТГ), ультразвукового исследования (УЗИ) шеи и сканирования всего тела с радиойодом. Именно после проведения комбинированного лечения ДРЩЖ (тотальной тиреоидэктомии и радиойодтерапии (РЙТ)) достигается максимальная диагностическая точность определения ТГ как маркера рецидива/персистенции заболевания. Влияние антител к тиреоглобулину (АТ-ТГ) может искажать результаты определения ТГ, приводя к занижению истинного уровня и ложноотрицательным ответам.
\end{abstract}

ЦЕЛЬ: сравнение прогностической ценности иммунометрических методов измерения ТГ с различной функциональной чувствительностью (ФС) для раннего выявления рецидива/персистенции ДРЩЖ.

ДИЗАЙН: в исследование были включены 76 больных ДРЩЖ после радикального лечения (тиреоидэктомия и РЙТ). Исходно все пациенты относились к группам очень низкого, низкого и промежуточного риска рецидива ( $\left.\mathrm{pT}_{1-3} \mathrm{~N}_{0-1 / 1} \mathrm{M}_{0}\right)$. Оценка отдаленных результатов лечения проводилась на основании данных о количестве рецидивов, развившихся за период исследования, срок наблюдения за больными - более 6 лет. Протокол наблюдения включал двукратное за год определение уровня базального ТГ (ТГб) на фоне терапии левотироксином (LT4), ежегодное проведение УзИ шеи, выполнение сцинтиграфии всего тела с радиойдом (CBTI, не менее трех раз за период наблюдения) с определением стимулированного уровня ТГ (ТГСТ) в ходе теста на стимуляцию продукции ТТГ. У $80 \%$ пациентов тесты на стимуляцию ТTГ выполнялись путем отмены приема LT4, в остальных случаях путем инъекции рекомбинантного ТТГ. Все образцы ТГ исследовали иммунометрическим методом с $Ф 4-0,9$ нг/мл и высокочувствительным методом с $Ф Ч \leq 0,2$ нг/мл (вч-ТГ). Каждое определение ТГ сопровождалось контролем титра АТ-ТГ.

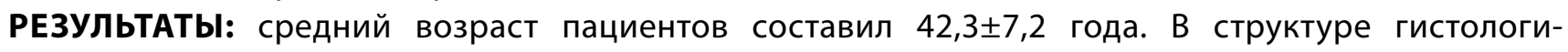
ческих типов ДРЩЖ преобладал папиллярный тип (94\%). Распространенность носительства АТ-ТГ составила 35,5\% ( $n=27)$. За первые 18 месяцев наблюдения у 11,8\% пациентов ( $\mathrm{n=9}$ ) выявлен рецидив ДРЩЖ на основании уровня ТГст (диапазон от 4,5 до 9,7 нг/мл) и был подтвержден визуализирующими методами (УЗИ и СВТІ); при этом, значения вч-ТГб находились в пределах 0,54-0,89 нг/мл. Наблюдение продолжено за 67 пациентами. В дальнейшем рецидив заболевания был выявлен у двух АТ-ТГ-положительных пациентов с уровнем ТГб и вч-ТГб менее 1,0 нг/мл (0,54 и 0,71 нг/мл соответственно). По итогам 5-летнего рубежа наблюдения доля рецидивов ДРЩЖ составила 14,5\% ( $\mathrm{n}=11)$. Методы с высокой ФЧ уверенно предсказывают (в 100\%) увеличение ТГст при отсутствии носительства АТ-ТГ. Для пациентов с неопределяемым уровнем ТГб и отсутствием АТ-ТГ отрицательный тест на стимуляцию ТТГ (ТГст $\leq 2,0$ нг/мл) был получен при уровне их вч-ТГб $\leq 0,4$ нг/мл. Методы со стандартной ФЧ, равно как и высокочувствительные тесты продемонстрировали низкую воспроизводимость результатов определения ТГб для всех АТ-ТГ-положительных пациентов, в то время как у АТ-ТГ-отрицательных пациентов все результаты были надежными. Не было выявлено корреляции между количественными значениями титра АТ-ТГ и уровня ТГб.

ВЫВоды: стимулированный ТГ остается надежным маркером рецидива ДРЩЖ. В условиях отсутствия АТ-ТГ для пациентов с подтверждённой клинической ремиссией заболевания (по результатам стТГ, СВТІ и УЗИ шеи через 12-18 месяцев от радикального комбинированного лечения) регулярное исследование вч-ТГб и УЗИ шеи являются достаточными инструментами для рутинного наблюдения. Высокочувствительные тесты имеют высокий потенциал в отношении раннего выявления рецидива 
заболевания у АТ-ТГ-негативных больных ДРЩЖ групп низкого и промежуточного риска, когда отрицательный результат определения вч-ТГб позволяет проводить СВТІ и определение ТГст с частотой согласно динамической стратификации рисков. Влияние АТ-ТГ значительно снижает диагностическое значение оценки ТГб иммунометрическими методами. При носительстве АТ-ТГ мониторинг вч-ТГб не может в полной мере заменить тест на стТГ, дополненный СВТІ.

В целом этот подход позволяет персонифицировать протокол динамического наблюдения за больными ДРЩЖ, значительно уменьшая потребность в избыточных исследованиях, повышая качество жизни пациентов и сокращая бремя затрат на лечение.

ФИНАНСИРОВАНИЕ: работа выполнена в рамках гранта Российского научного фонда (РНФ) №17-7530035. 